\title{
Dynamics of Modified Chaplygin Gas in Brane World Scenario: Phase Plane Analysis
}

\author{
Prabir Rudra $1_{*}$ \\ Ujjal Debnath $*$ \\ Ritabrata Biswas 3 \\ *Department of Mathematics, Bengal Engineering and Science University, Shibpur, Howrah-711 103, India. \\ $\dagger$ Department of Mathematics, Jadavpur University, Kolkata-700 032, India.
}

\begin{abstract}
In this work we investigate the background dynamics when dark energy is coupled to dark matter with a suitable interaction in the universe described by brane cosmology. Here DGP and the RSII brane models have been considered separately. Dark energy in the form of modified Chaplygin gas is considered. A suitable interaction between dark energy and dark matter is considered in order to at least alleviate (if not solve) the cosmic coincidence problem. The dynamical system of equations is solved numerically and a stable scaling solution is obtained. A significant attempt towards the solution of the cosmic coincidence problem is taken. The statefinder parameters are also calculated to classify the dark energy models. Graphs and phase diagrams are drawn to study the variations of these parameters. It is also seen that the background dynamics of modified Chaplygin gas is completely consistent with the notion of an accelerated expansion in the late universe. Finally, it has been shown that the universe in both the models follows the power law form of expansion around the critical point, which is consistent with the known results.
\end{abstract}

\section{Introduction}

The idea of the static universe once conceived by Albert Einstein faced a big threat when at the turn of the last century observations of Ia Supernova confirmed that our universe is suffering from an accelerated expansion (Perlmutter, S. et al., 1999; Spergel, D. N. et al.2003). Now belief and observations are always to be supported by some model. In the quest of finding a suitable model for universe, Cosmologists started to investigate the root cause that is triggering this expansion. Fundamentally, we were to modify Einstein's equation either by modifying the left hand side, i.e., modifying the idea of Einstein gravity or to modify the right hand side which immediately speculate the nature of the matter inside the universe. If our Universe is filled up by some invisible fluid causing a negative pressure then it violates the strong energy condition i.e. $\rho+3 p<0$. Because of its invisible nature this energy component was aptly termed as dark energy (DE) (Riess, A. G. et al., 2004).

With the introduction of $\mathrm{DE}$, the search began for different candidates that can effectively play the role of DE. DE represented by a scalar field 4 is often called quintessence. Not only scalar field but also there are other Dark fluid models like Chaplygin gas which plays the role of DE very efficiently. The earliest form of this was known as pure Chaplygin gas (Kamenshchik, A. et al.,2001; Gorini, V. et al. 2004). Extensive research saw pure Chaplygin gas first modify into generalized Chaplygin gas (Gorini, V. et al., 2006; Alam, U. et al. 2003; Bento, M. C. et al. 2002; Carturan, D. and Finelli, F. 2003; Barreiro, T. and Sen, A.A. 2004) and later into modified Chaplygin gas (MCG) obeying the equation of state (Benaoum, H. B., 2002; Debnath, U., 2004)

$$
p=A \rho-\frac{B}{\rho^{\alpha}},
$$

where $p$ and $\rho$ are respectively the pressure and energy density and $0 \leq \alpha \leq 1, A$ and $B$ are positive constants. Currently, we live in a special epoch where the densities of DE and DM are comparable. Although they have evolved

\footnotetext{
${ }^{1}$ prudra.math@gmail.com

2ujjal@iucaa.ernet.in, ujjaldebnath@yahoo.com

${ }^{3}$ biswas.ritabrata@gmail.com

${ }^{4}$ in the presence of a scalar field the transition from a universe filled with matter to an exponentially expanding universe is justified
} 
independently from different mass scales. This is known as the famous cosmic coincidence problem. till date several attempts have been made to find a solution to this problem (del Campo, S. et al 2009; Leon, G. and Saridakis, E.N. 2010; Jimenez, J.B. and Maroto, A.L.2008; Berger, M.S. and Shojae, H. 2006; Zhang, X. 2005; Griest, K. 2002; Jamil, M. and Rehman, F. 2009; Jamil, M. et al. 2010a; Jamil, M. and Saridakis, E. N. 2010b; Jamil, M. and Farooq, M. U., 2010c; Jamil, M. et al. 2010d). A suitable interaction between DE and DM is required if we wish to find an effective solution to this problem. It is obvious that there has been a transition from a matter dominated universe to dark energy dominated universe, by exchange of energy at an appropriate rate. Now the expansion history of the universe as determined by the supernovae and CMB data (Jamil, M.et al. 2010a; Jamil, M. and Saridakis, E. N. 2010b) bounds us to fix the decay rate such that it is proportional to the present day Hubble parameter. Keeping the fact in mind cosmologists all over the world have studied and proposed a variety of interacting DE models (Setare, M. R. 2006,2007c; Hu, B. and Ling, Y. 2006; Wu, P. and Yu, H. 2007; Jamil, M. 2010e, 2011; Dalal, N. 2001).

Here we shall use MCG as the DE and the equation of state for MCG is given by (11) with $\rho=\rho_{m c g}$ Recent developments regarding MCG can be found in (Lu, J. et al. 2008; Dao-Jun, L., Xin-Zhou, L. 2005; Jing, H. et. al. 2008; Debnath, U. 2007). This special form also appears to be consistent with the WMAP 5-year data and henceforth support the unified model with DE and DM based on generalized CG (Makler, M. et al. 2003; Setare, M.R. 2007a, 2007b, 2009; Barreiro, T. et al. 2008). Recent supernovae data also favours the two-fluid cosmological model with CG and matter (Panotopoulos, G. 2008).

As we have stated earlier, modifying the right hand side of Einstein's equation was not the only way to explain the increase in the rate of the expansion, rather to modify the gravity part of the left hand side is also to demonstrate the present day universe. In this branch Brane-gravity was introduced and brane cosmology was developed. A review on brane-gravity and its various applications with special attention to cosmology is available in (Rubakov, V. A. 2001; Maartens, R. 2004; Brax. P. et al. 2004; Csa ki, C. 2004). In this work we consider the two most popular brane models, namely DGP and RS II branes. Our main aim of this work is to examine the nature of the different physical parameters for the universe around the stable critical points in two brane world models in presence of MCG. Impact of any future singularity caused by the DE in brane world models will be studied.

This paper is organized as follows: Section 2 comprises of the dynamical system analysis in DGP brane model. Section 3 deals with the dynamical system analysis in RS II brane model, followed by a general discussion in section 4.

\section{Dynamical system analysis in DGP brane world model}

A simple and effective model of brane-gravity is the Dvali-Gabadadze-Porrati (DGP) braneworld model (Dvali, G. R. et al. 2000; Deffayet, D. 2001; Deffayet, D. et al. 2002) which models our 4-dimensional world as a FRW brane embedded in a 5-dimensional Minkowski bulk. It explains the origin of DE as the gravity on the brane leaking to the bulk at large scale. On the 4-dimensional brane the action of gravity is proportional to $M_{p}^{2}$ whereas in the bulk it is proportional to the corresponding quantity in 5-dimensions. The model is then characterized by a cross over length scale $r_{c}=\frac{M_{p}^{2}}{2 M_{5}^{2}}$ such that gravity is 4-dimensional theory at scales $a<<r_{c}$ where matter behaves as pressureless dust, but gravity leaks out into the bulk at scales $a>>r_{c}$ and matter approaches the behaviour of a cosmological constant. Moreover it has been shown that the standard Friedmann cosmology can be firmly embedded in DGP brane.

It may be noted that in literature, standard DGP model has been generalized to (i) LDGP model by adding a cosmological constant (Lue,A. and Starkman,G. D. 2004), (ii) QDGP model by adding a quintessence perfect fluid (Chimento,L. P. et al. 2006), (iii) CDGP model by Chaplygin gas (Bouhmadi-Lopez, M. and Lazkoz, R. 2007) and (iv) SDGP by a scalar field (Zhang, H. and Zhu, Z. H. 2007). In (Wu, X. et al. 2008) the DGP model has been analysed by adding Holographic DE (HDE).

\subsection{Basic equations in DGP brane model}

While flat, homogeneous and isotropic brane is being considered, the Friedmann equation in DGP brane model(Dvali, G. R. et al, 2000; Deffayet, D., 2001; Deffayet, D. et al, 2002) is modified to the equation

$$
H^{2}=\left(\sqrt{\frac{\rho}{3}+\frac{1}{4 r_{c}^{2}}}+\epsilon \frac{1}{2 r_{c}}\right)^{2},
$$

where $H=\frac{\dot{a}}{a}$ is the Hubble parameter, $\rho$ is the total cosmic fluid energy density and $r_{c}=\frac{M_{p}^{2}}{2 M_{5}^{2}}$ is the cross-over scale which determines the transition from $4 \mathrm{D}$ to $5 \mathrm{D}$ behaviour and $\epsilon= \pm 1$ (choosing $M_{p}^{2}=8 \pi G=1$ ). For $\epsilon=+1$, we 
have standard DGP $(+)$ model which is self accelerating model without any form of DE, and effective $w$ is always non-phantom. However for $\epsilon=-1$, we have DGP $(-)$ model which does not self accelerate but requires DE on the brane. It experiences $5 \mathrm{D}$ gravitational modifications to its dynamics which effectively screen DE. Brane world scenario is actually a modified gravity theory. If we write the Einstein equation for brane world in terms of Einstein gravity then the extra term can be treated as the effective DE But that is not the physical DE. Moreover this DE is applicable only in Einstein gravity. But here we will consider the physical DE in brane world. So we have introduced the MCG type fluid in brane. There are few many candidates of DE models, such as scalar field (Nojiri, S.and Oditsov, S.D. 2004), phantom (Nojiri, S. et al 2005), k-essence (Bamba, K.et al 2011), tachyonic field ((Nojiri, S.and Oditsov, S.D. 2003 ), etc. MCG being a modified form of DE with a unique equation of state for DE and DM. Also MCG is the observational viable model (Thakur, P. et al 2009, Dev, A. et al 2003). It interpolates between radiation in early stage and $\Lambda \mathrm{CDM}$ in late stage.

As in the present problem the interaction between DE and pressureless dark matter has been taken into account for interacting DE and DM the energy balance equation will be

$$
\begin{gathered}
\dot{\rho}_{m c g}+3 H\left(1+w_{m c g}\right) \rho_{m c g}=-Q, \text { for } M C G \text { and } \\
\dot{\rho}_{m}+3 H \rho_{m}=Q, \text { for the DM interacting with } M C G .
\end{gathered}
$$

where $Q=3 b H \rho$ is the interaction term, $b$ is the coupling parameter (or transfer strength) and $\rho=\rho_{m c g}+\rho_{m}$ is the total cosmic energy density which satisfies the energy conservation equation $\dot{\rho}+3 H(\rho+p)=0$ (Guo, Z.-K. and Zhang, Y.-Z. 2005; delCampo, S. et. al. 2008).

As we have lack of information about the fact how do DE and DM interact so we are not able to esteemate the interaction term from the first principles. However, the negativity of $Q$ immediately implies the possibility of having negative DE in the early universe which is overruled by to the necessity of the second law of thermodynamics to be held(Alcaniz, J. S. and Lima, J. A. S. 2005). Hence $Q$ must be positive and small. From the observational data of 182 Gold type Ia supernova samples, CMB data from the three year WMAP survey and the baryonic acoustic oscillations from the Sloan Digital Sky Survey, it is estimated that the coupling parameter between DM and DE must be a small positive value (of the order of unity), which satisfies the requirement for solving the cosmic coincidence problem and the second law of thermodynamics (Feng, C. et. al. 2008). Due to the underlying interaction, the beginning of the accelerated expansion is shifted to higher redshifts. Consequently using the Friedmann equation (2) and the conservation equation, we obtain the modified Raychaudhuri equation

$$
\left(2 H-\frac{\epsilon}{r_{c}}\right) \dot{H}=-H(\rho+p)
$$

\subsection{Dynamical system analysis}

In this subsection we plan to analyse the dynamical system. For that firstly the conversion of the physical parameters into some dimensionless form, given by

$$
x=\ln a, \quad u=\frac{\rho_{m c g}}{3 H^{2}}, \quad v=\frac{\rho_{m}}{3 H^{2}}
$$

where the present value of the scale factor $a_{0}=1$ is assumed. Inclusion of (11) into (6) gives us the parameter gradients as

$$
\frac{d u}{d x}=-3 b(u+v)-3 u\left(1+w_{m c g}^{(D G P)}\right)+\frac{3 u\left\{\epsilon-\sqrt{1+\left(\epsilon^{2}-1\right)(u+v)}\right\}\left\{u\left(1+w_{m c g}^{(D G P)}\right)+v\right\}}{\epsilon(u+v)-\sqrt{1+\left(\epsilon^{2}-1\right)(u+v)}}
$$

and

$$
\frac{d v}{d x}=3 b(u+v)-3 v+\frac{3 v\left\{\epsilon-\sqrt{1+\left(\epsilon^{2}-1\right)(u+v)}\right\}\left\{u\left(1+w_{m c g}^{(D G P)}\right)+v\right\}}{\epsilon(u+v)-\sqrt{1+\left(\epsilon^{2}-1\right)(u+v)}} .
$$

Where, $w_{m c g}^{(D G P)}$ is the EoS parameter for MCG determined as

$$
w_{m c g}^{(D G P)}=\frac{p_{m c g}}{\rho_{m c g}}=A-B\left[\frac{2^{2} r_{c}^{2}(1-u-v)^{2}}{3 u\left(\epsilon-\sqrt{1+\left(\epsilon^{2}-1\right)(u+v)}\right)^{2}}\right]^{(\alpha+1)} .
$$




\subsubsection{Critical point}

For mathematical simplicity, we consider $\alpha=1$ only. The critical points of the above system are obtained by putting

$\frac{d u}{d x}=0=\frac{d v}{d x}$. Now this system of equation does not yield an explicit solution. So we have to put some special values to the constants which will yield a non zero positive solution of the system of equations. Considering $A=0$ and $\epsilon=1$ we obtain the following critical value to the system.

$$
\begin{gathered}
u_{c}=1-b-\frac{3 D}{2 B r^{4}}-\frac{\sqrt{3}}{2} \sqrt{\frac{3\left(1-3 b+3 b^{2}-b^{3}\right)}{B r^{4}}-\frac{4\left(1+4 b-6 b^{2}+4 b^{3}-b^{4}\right)}{D}} \\
v_{c}=\frac{1}{B r^{4}}\left(\frac{9-18 b+9 b^{2}}{u_{c}}+\frac{3}{2} D\right)+3+b+\frac{\sqrt{3 N}}{2}-\frac{1+3 b-3 b^{2}+b^{3}}{u_{c}^{3}}+\frac{4\left(1-2 b+b^{2}\right)}{u_{c}^{2}}-\frac{6(1+b)}{u_{c}}
\end{gathered}
$$

where

$$
D=\sqrt{B\left(1-3 b+3 b^{2}-b^{3}\right)} r^{2}
$$

and

$$
N=\left\{\frac{2}{\sqrt{3}}\left(u_{c}+b-1+\frac{3 \sqrt{1-3 b+3 b^{2}-b^{3}}}{2 \sqrt{B} r^{2}}\right)\right\}^{2}
$$

The critical point correspond to the era dominated by DM and MCG type DE. For the critical point $\left(u_{c}, v_{c}\right)$, the equation of state parameter (9) of the interacting DE takes the form

$$
w_{m c g}^{(D G P)}=A-\frac{B 2^{2(\alpha+1)} r_{c}^{2(\alpha+1)}\left(1-u_{c}-v_{c}\right)^{2(\alpha+1)}}{3^{\alpha+1} u_{c}^{\alpha+1}\left(\epsilon-\sqrt{1+\left(\epsilon^{2}-1\right)\left(u_{c}+v_{c}\right)}\right)^{2(\alpha+1)}} .
$$

\subsubsection{Stability around critical point}

Now we check the stability of the dynamical system (eqs. (7) and (8)) about the critical point. In order to do this, we linearize the governing equations about the critical point i.e.,

$$
u=u_{c}+\delta u \text { and } v=v_{c}+\delta v,
$$

Now if we assume $f=\frac{d u}{d x}$ and $g=\frac{d v}{d x}$, then we may obtain

$$
\delta\left(\frac{d u}{d x}\right)=\left[\partial_{u} f\right]_{c} \delta u+\left[\partial_{v} f\right]_{c} \delta v
$$

and

$$
\delta\left(\frac{d v}{d x}\right)=\left[\partial_{u} g\right]_{c} \delta u+\left[\partial_{v} g\right]_{c} \delta v
$$

where

$$
\begin{gathered}
\partial_{u} f=\left[-3 b-3 B u\left(\frac{-32 \theta^{4}\left(\epsilon^{2}-1\right) r_{c}^{4}}{9 u^{2} K(\epsilon-K)^{5}}+\frac{64 \theta^{3} r_{c}^{4}}{9 u^{2}(\epsilon-K)^{4}}+\frac{32 \theta^{4} r_{c}^{4}}{9 u^{3}(\epsilon-K)^{4}}\right)-3\left(1+A-\frac{16 B \theta^{4} r_{c}^{4}}{9 u^{2}(\epsilon-K)^{4}}\right)\right. \\
+\frac{1}{\epsilon(u+v)-3 u K(\epsilon-K)}\left[1+A-\frac{16 B \theta^{4} r_{c}^{4}}{9 u^{2}(\epsilon-K)^{4}}+u\left(-\frac{32 B \theta^{4}\left(\epsilon^{2}-1\right) r_{c}^{4}}{9 u^{2} K(\epsilon-K)^{5}}+\frac{64 B \theta^{3} r_{c}^{4}}{9 u^{2}(\epsilon-K)^{4}}+\frac{32 B \theta^{4} r_{c}^{4}}{9 u^{3}(\epsilon-K)^{4}}\right)\right] \\
-\frac{3 u\left(\epsilon-\frac{\epsilon^{2}-1}{2 K}\right)(\epsilon-K)\left(v+u\left(1+A-\frac{16 B \theta^{4} r_{c}^{4}}{9 u^{2}(\epsilon-K)^{4}}\right)\right)}{((u+v) \epsilon-K)^{2}}-\frac{3 u\left(\epsilon^{2}-1\right)\left(v+u\left(1+A-\frac{16 B \theta^{4} r_{c}^{4}}{9 u^{2}(\epsilon-K)^{4}}\right)\right)}{2 K((u+v) \epsilon-K)} \\
\left.+\frac{3(\epsilon-K)\left(v+u\left(1+A-\frac{16 B \theta^{4} r_{c}^{4}}{9 u^{2}(\epsilon-K)^{4}}\right)\right)}{(u+v) \epsilon-K}\right]
\end{gathered}
$$




$$
\begin{aligned}
& \partial_{v} f=\left[-3 b-3 u\left(-\frac{32 B \theta^{4}\left(\epsilon^{2}-1\right) r_{c}^{4}}{9 u^{2} K(\epsilon-K)^{5}}+\frac{64 B \theta^{3} r_{c}^{4}}{9 u^{2}(\epsilon-K)^{4}}\right)+\frac{3 u(\epsilon-K)\left(1+u\left(-\frac{32 B \theta^{4}\left(\epsilon^{2}-1\right) r_{c}^{4}}{9 u^{2} K(\epsilon-K)^{5}}+\frac{64 B \theta^{3} r_{c}^{4}}{9 u^{2}(\epsilon-K)^{4}}\right)\right)}{(u+v) \epsilon-K}\right. \\
& \left.-\frac{3 u\left(\epsilon-\frac{\epsilon^{2}-1}{2 K}\right)(\epsilon-K)\left(v+u\left(1+A-\frac{16 B \theta^{4} r_{c}^{4}}{9 u^{2}(\epsilon-K)^{4}}\right)\right)}{((u+v) \epsilon-K)^{2}}-\frac{3 u\left(\epsilon^{2}-1\right)\left(v+u\left(1+A-\frac{16 B \theta^{4} r_{c}^{4}}{9 u^{2}(\epsilon-K)^{4}}\right)\right)}{2 K((u+v) \epsilon-K)}\right] \\
& \partial_{u} g=\left[3 b+\frac{1}{\epsilon(u+v)-3 v K(\epsilon-K)}\left[1+A-\frac{16 B \theta^{4} r_{c}^{4}}{9 u^{2}(\epsilon-K)^{4}}+u\left(-\frac{32 B \theta^{4}\left(\epsilon^{2}-1\right) r_{c}^{4}}{9 u^{2} K(\epsilon-K)^{5}}+\frac{64 u B \theta^{3} r_{c}^{4}+32 B \theta^{4} r_{c}^{4}}{9 u^{3}(\epsilon-K)^{4}}\right)\right]\right. \\
& \left.-\frac{3 v\left(\epsilon-\frac{\epsilon^{2}-1}{2 K}\right)(\epsilon-K)\left(v+u\left(1+A-\frac{16 B \theta^{4} r_{c}^{4}}{9 u^{2}(\epsilon-K)^{4}}\right)\right)}{((u+v) \epsilon-K)^{2}}-\frac{3 v\left(\epsilon^{2}-1\right)\left(v+u\left(1+A-\frac{16 B \theta^{4} r_{c}^{4}}{9 u^{2}(\epsilon-K)^{4}}\right)\right)}{2 K((u+v) \epsilon-K)}\right] \\
& \partial_{v} g=\left[-3+3 b+\frac{3 v(\epsilon-K)\left[1+u\left(-\frac{32 B \theta^{4}\left(\epsilon^{2}-1\right) r_{c}^{4}}{9 u^{2} K(\epsilon-K)^{5}}+\frac{64 B \theta^{3} r_{c}^{4}}{9 u^{2}(\epsilon-K)^{4}}\right)\right]}{(u+v) \epsilon-K}+\frac{3(\epsilon-K)\left(v+u\left(1+A-\frac{16 B \theta^{4} r_{c}^{4}}{9 u^{2}(\epsilon-K)^{4}}\right)\right)}{(u+v) \epsilon-K}\right. \\
& \left.-\frac{3 v(\epsilon-K)}{((u+v) \epsilon-K)^{2}}\left(\epsilon-\frac{\left(\epsilon^{2}-1\right)}{2 K}\right)\left(v+u\left(1+A-\frac{16 B \theta^{4} r_{c}^{4}}{9 u^{2}(\epsilon-K)^{4}}\right)\right)-\frac{3 v\left(\epsilon^{2}-1\right)\left(v+u\left(1+A-\frac{16 B \theta^{4} r_{c}^{4}}{9 u^{2}(\epsilon-K)^{4}}\right)\right)}{2 K((u+v) \epsilon-K)}\right]
\end{aligned}
$$

where $K=\sqrt{1+(u+v)\left(\epsilon^{2}-1\right)}, \quad$ and $\quad \theta=(1-u-v)$. The subscript $c$ refers to quantities evaluated at the critical point of the dynamical system.

\subsubsection{Nature of cosmological parameters}

We calculate the deceleration parameter $q=-1-\left(\dot{H} / H^{2}\right)$, in this model as,

$$
q^{(D G P)}=-1+\frac{3}{2}\left\{\frac{12 r_{c}^{2}\left(1+w_{m c g}^{(D G P)} \frac{\rho_{m c g}}{\rho}\right)}{\sqrt{4 r_{c}^{2}+3 \sigma}\left(\sqrt{4 r_{c}^{2}+3 \sigma}+\frac{\epsilon}{\sqrt{\sigma}}\right)}\right\},
$$

where $\sigma=\frac{1}{\rho}$. The above deceleration parameter can be written in terms of dimensionless density parameter $\Omega_{m c g}=$ $\frac{\rho_{m c g}}{\rho}$ as

$$
q^{(D G P)}=-1+\frac{3}{2}\left\{\frac{12 r_{c}^{2}\left(1+w_{m c g}^{(D G P)} \Omega_{m c g}\right)}{\sqrt{4 r_{c}^{2}+3 \sigma}\left(\sqrt{4 r_{c}^{2}+3 \sigma}+\frac{\epsilon}{\sqrt{\sigma}}\right)}\right\} .
$$

Now using (6) we obtain $\Omega_{m c g}=\frac{\rho_{m c g}}{\rho}=\frac{u}{u+v}$. So from equation (21) it is obvious that,

$$
q^{(D G P)}=-1+\frac{3}{2}\left\{\frac{12 r_{c}^{2}\left(1+\frac{u w_{m c g}^{(D G P)}}{u+v}\right)}{\sqrt{4 r_{c}^{2}+3 \sigma}\left(\sqrt{4 r_{c}^{2}+3 \sigma}+\frac{\epsilon}{\sqrt{\sigma}}\right)}\right\}
$$

The solution corresponding to the stable critical point, such that $(u, v) \rightarrow\left(u_{c}, v_{c}\right)$. Hence using (22) we get

$$
q_{c}^{(D G P)}=-1+\frac{3}{2} X_{(D G P)}, \text { where } X_{(D G P)}=\frac{12 r_{c}^{2}\left(1+\frac{w_{m c g}^{(D G P)} u_{c}}{u_{c}+v_{c}}\right)}{\left\{\sqrt{4 r_{c}^{2}+3 \sigma}\left(\sqrt{4 r_{c}^{2}+3 \sigma}+\frac{\epsilon}{\sqrt{\sigma}}\right)\right\}} .
$$




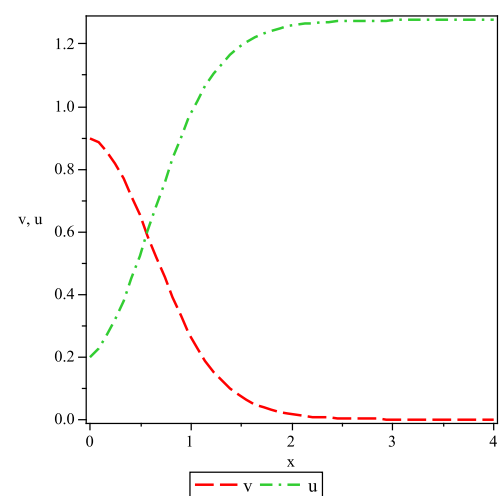

Fig. 1

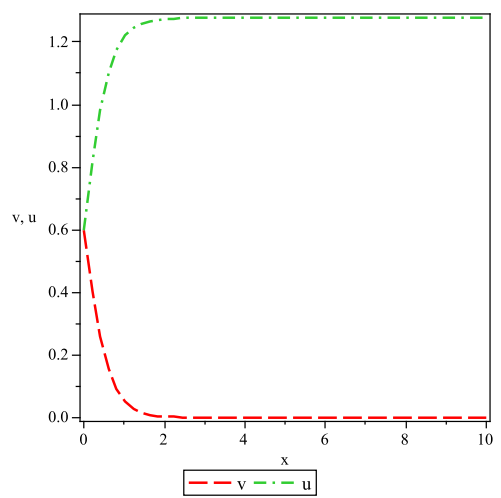

Fig. 2

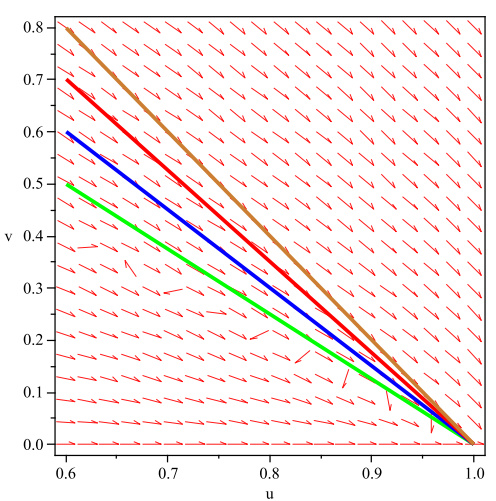

Fig. 3

Fig 1 : The dimensionless density parameters are plotted against e-folding time. The initial condition is $v(0)=0.9, u(0)=0.2$. Other parameters are fixed at $\alpha=1, b=0, A=1, B=0.5$ and $r_{c}=10$.

Fig 2: The dimensionless density parameters are plotted against e-folding time. The initial condition is $v(0)=0.6, u(0)=0.6$. The other parameters are fixed at $\alpha=1, b=0, A=1, B=0.5$ and $r_{c}=10$.

Fig 3 : The phase diagram of the parameters depicting an attractor solution. The initial conditions chosen are $v(0)=0.5, u(0)=0.6$ (green); $v(0)=0.6, u(0)=0.6$ (blue); $v(0)=0.7, u(0)=0.6$ (red); $v(0)=0.8, u(0)=0.6$ (brown). Other parameters are fixed at $\alpha=1, b=0, A=0.3, B=1$ and $r_{c}=10000$.

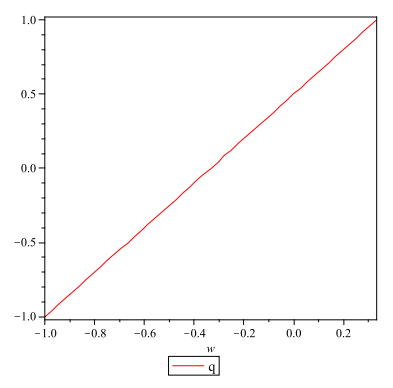

Fig. 4

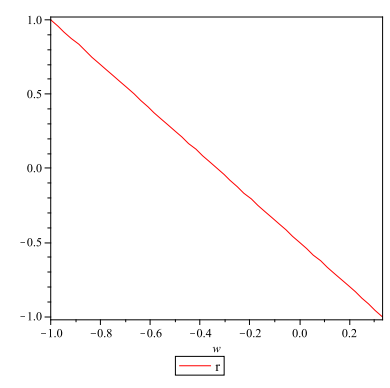

Fig. 5

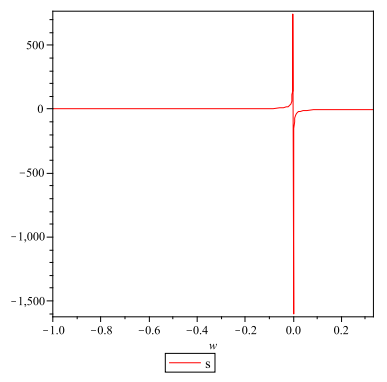

Fig. 6

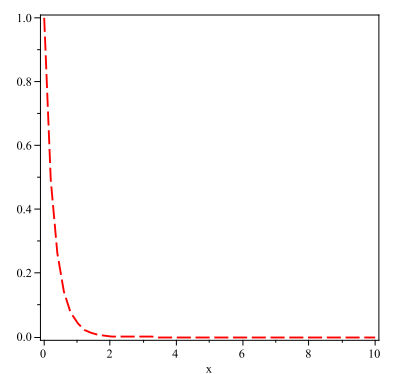

Fig. 7

Fig 4 : The deceleration parameter is plotted against the state parameter. Other parameters are fixed at $B=1, b=0, r_{c}=10000, \epsilon=-1$.

Fig 5: The statefinder parameter $r$ is plotted against the state parameter. The other parameters are fixed at $B=1, b=0, r_{c}=10000, \epsilon=-1$.

Fig 6 : The statefinder parameter $s$ is plotted against the state parameter. The other parameters are fixed at $B=1, b=0, r_{c}=10000, \epsilon=-1$.

Fig 7 : The ratio of density parameters is shown against e-folding time. The initial conditions chosen are $\mathrm{v}(0)=0.6, \mathrm{u}(0)=0.6$. The other parameters are fixed at $\alpha=1, A=1, b=0, B=0.5, r_{c}=10$. 
Moreover the Hubble parameter can be obtained as

$$
H=\frac{2}{3 X_{(D G P)} t}
$$

where we have ignored the integration constant. Integration of (24) yields

$$
a(t)=a_{0} t^{\frac{2}{3 X_{(D G P)}}}
$$

which gives a power law form of the expansion. In order to have accelerating universe $\frac{2}{3 X_{(D G P)}}>1$ i.e., $0<$ $X_{(D G P)}<\frac{2}{3}$. Using this range of $X_{(D G P)}$ in the equation $q_{c}^{(D G P)}=-1+\frac{3}{2} X_{(D G P)}$. We get the range of $q_{c}^{(D G P)}$ as $-1<q_{c}^{(D G P)}<0$. Therefore the deceleration parameter is negative and hence the result is consistent with the fact that the universe is undergoing an accelerated expansion.

Sahni et al (2003) introduced a pair of cosmological diagnostic pair $\{r, s\}$ which is known as as statefinder parameters. The two parameters are dimensionless and are geometrical since they are derived from the cosmic scale factor alone. Also this pair generalizes the well-known geometrical parameters like the Hubble parameter and the deceleration parameter. The statefinder parameters are given by

$$
r \equiv \frac{\dddot{a}}{a H^{3}}, \quad s \equiv \frac{r-1}{3(q-1 / 2)} .
$$

In the DGP brane model, we have the following expressions of $r$ and $s$ as

$$
r_{(D G P)}=\left(1-\frac{3 X_{(D G P)}}{2}\right)\left(1-3 X_{(D G P)}\right) .
$$

and

$$
s_{(D G P)}=X_{(D G P)} .
$$

\section{Dynamical system analysis for RS II brane world}

Randall and Sundrum (1999a, 1999b) proposed a bulk-brane model to explain the higher dimensional theory, popularly known as RS II brane model. According to this model we live in a four dimensional world (called 3-brane, a domain wall) which is embedded in a 5D space time (bulk). All matter fields are confined in the brane whereas gravity can only propagate in the bulk. The consistency of this brane model with the expanding universe has given popularity to this model of late in the field of cosmology.

\subsection{Basic equations in RS II brane model}

In RS II model the effective equations of motion on the 3-brane embedded in $5 \mathrm{D}$ bulk having $Z_{2}$-symmetry are given by [Maartens, R. 2000, 2004; Randall and Sundrum, 1999b; Shiromizu et al, 2000; Maeda et al, 2000; Sasaki et al, 2000]

$$
{ }^{(4)} G_{\mu \nu}=-\Lambda_{4} q_{\mu \nu}+\kappa_{4}^{2} \tau_{\mu \nu}+\kappa_{5}^{4} \Pi_{\mu \nu}-E_{\mu \nu}
$$

where

$$
\begin{gathered}
\kappa_{4}^{2}=\frac{1}{6} \lambda \kappa_{5}^{4}, \\
\Lambda_{4}=\frac{1}{2} \kappa_{5}^{2}\left(\Lambda_{5}+\frac{1}{6} \kappa_{5}^{2} \lambda^{2}\right)
\end{gathered}
$$

and

$$
\Pi_{\mu \nu}=-\frac{1}{4} \tau_{\mu \alpha} \tau_{\nu}^{\alpha}+\frac{1}{12} \tau \tau_{\mu \nu}+\frac{1}{8} q_{\mu \nu} \tau_{\alpha \beta} \tau^{\alpha \beta}-\frac{1}{24} q_{\mu \nu} \tau^{2}
$$

and $E_{\mu \nu}$ is the electric part of the 5D Weyl tensor. Here $\kappa_{5}, \Lambda_{5}, \tau_{\mu \nu}$ and $\Lambda_{4}$ are respectively the $5 \mathrm{D}$ gravitational coupling constant, 5D cosmological constant, the brane tension (vacuum energy), brane energy-momentum tensor and effective 4D cosmological constant. The explicit form of the above modified Einstein equations in flat universe are 


$$
3 H^{2}=\Lambda_{4}+\kappa_{4}^{2} \rho+\frac{\kappa_{4}^{2}}{2 \lambda} \rho^{2}+\frac{6}{\lambda \kappa_{4}^{2}} \mathcal{U}
$$

and

$$
2 \dot{H}+3 H^{2}=\Lambda_{4}-\kappa_{4}^{2} p-\frac{\kappa_{4}^{2}}{2 \lambda} \rho p-\frac{\kappa_{4}^{2}}{2 \lambda} \rho^{2}-\frac{2}{\lambda \kappa_{4}^{2}} \mathcal{U}
$$

The dark radiation $\mathcal{U}$ obeys

$$
\dot{\mathcal{U}}+4 H \mathcal{U}=0
$$

where $\rho=\rho_{m c g}+\rho_{m}$ and $p=p_{m c g}+p_{m}$ are the total energy density and pressure respectively. The continuity equations for dark energy and dark matter are given in (3) and (4). Now we shall study the dynamical system assuming $\Lambda_{4}=\mathcal{U}=0$ (in absence of cosmological constant and dark radiation).

\subsection{Dynamical system analysis}

Now to analyze the dynamical system we define three variables $x, u$ and $v$ as given in equation (6). Now making use of (3), (4), (6), (33) and (34) we get,

$\frac{d u}{d x}=-3 b(u+v)-3 u\left(1+w_{m c g}^{(R S I I)}\right)+3 \kappa_{4}^{2} u\left\{u\left(1+w_{m c g}^{(R S I I)}\right)+v\right\}-\frac{3 u}{u+v}\left\{1-\kappa_{4}^{2}(u+v)\right\}\left\{u\left(2+w_{m c g}^{(R S I I)}\right)+2 v\right\}$

and

$$
\frac{d v}{d x}=3 b(u+v)-3 v+3 v\left[\kappa_{4}^{2}\left\{u\left(1+w_{m c g}^{(R S I I)}\right)+v\right\}-\frac{3}{u+v}\left\{1-\kappa_{4}^{2}(u+v)\right\}\left\{u\left(2+w_{m c g}^{(R S I I)}\right)+2 v\right\}\right]
$$

where

$$
w_{m c g}^{(R S I I)}=A-\left[\frac{B \kappa_{4}^{2}(u+v)^{2}}{2 u \lambda\left\{1-\kappa_{4}^{2}(u+v)\right\}}\right]^{\alpha+1}
$$

\subsubsection{Critical point}

Similar to DGP brane model, the critical points of the above system are obtained by putting $\frac{d u}{d x}=0=\frac{d v}{d x}$. Now this system of equation does not yield an explicit solution. So we have to put some special values to the constants which will yield non-zero positive solution of the system of equations. Considering $A=0, B=1, b=0.5, \lambda=1, \alpha=1$ and $\kappa=1$ we obtain the following critical points to the system.

$$
\begin{array}{ll}
u_{1 c}=0.491891, & v_{1 c}=0.190983 \\
u_{2 c}=1.19631, & v_{2 c}=0.836396 \\
u_{3 c}=2.22826, & v_{3 c}=10.9091
\end{array}
$$

\subsubsection{Stability around critical point}

Now if we write $\tilde{f}=\frac{d u}{d x}$ and $\tilde{g}=\frac{d v}{d x}$, then we can obtain the following expressions

$$
\delta\left(\frac{d u}{d x}\right)=\left[\partial_{u} \tilde{f}\right]_{c} \delta u+\left[\partial_{v} \tilde{f}\right]_{c} \delta v
$$

and

$$
\delta\left(\frac{d v}{d x}\right)=\left[\partial_{u} \tilde{g}\right]_{c} \delta u+\left[\partial_{v} \tilde{g}\right]_{c} \delta v
$$

where

$$
\partial_{u} \tilde{f}=\frac{3}{4 u^{2}(u+v)^{2}\left\{(u+v) k^{2}-1\right\}^{3} \lambda^{2}}\left[B(u+v)^{4} k^{4}\left\{-6 u^{2}-2 u v+v^{2}+(u+v)\left(10 u^{2}-v^{2}\right) k^{2}-4 u^{2}(u+v)^{2} k^{4}\right\}\right.
$$




$$
\begin{gathered}
\left.+4 u^{2}\left\{(u+v) k^{2}-1\right\}^{3}\left\{-3 u^{2}-2 A u^{2}-b u^{2}-6 u v-4 A u v-2 b u v-3 v^{2}-A v^{2}-b v^{2}+(u+v)^{2}((6+4 A) u+3 v) k^{2}\right\} \lambda^{2}\right] \\
\partial_{v} \tilde{f}=\frac{3}{4 u(u+v)^{2}\left\{(u+v) k^{2}-1\right\}^{3} \lambda^{2}}\left[B(u+v)^{4} k^{4}\left\{-7 u-4 v+(u+v)(11 u+2 v) k^{2}-4 u(u+v)^{2} k^{4}\right\}\right. \\
\left.+4 u\left\{(u+v) k^{2}-1\right\}^{3}\left\{A u^{2}+(u+v)^{2}\left(-b+3 u k^{2}\right)\right\} \lambda^{2}\right] \\
\partial_{u} \tilde{g}=3\left[b+v\left\{(7+4 A) k^{2}-\frac{3 A v}{(u+v)^{2}}\right\}+\frac{B v(u+v)^{2} k^{4}\left\{-6 u+3 v+(12 u-7 v)(u+v) k^{2}+4(v-u)(u+v)^{2} k^{4}\right\}}{4 u^{2}\left\{(u+v) k^{2}-1\right\}^{3} \lambda^{2}}\right] \\
\partial_{v} \tilde{g}=\frac{3}{4 u(u+v)^{2}\left\{(u+v) k^{2}-1\right\}^{3} \lambda^{2}}\left[B(u+v)^{4} k^{4}\left\{-3(u+4 v)+(u+v)(7 u+26 v) k^{2}-4(u+v)^{2}(u+3 v) k^{4}\right\}\right. \\
\left.+4 u\left\{(u+v) k^{2}-1\right\}^{3}\left\{-7 u^{2}-3 A u^{2}+b u^{2}-14 u v+2 b u v-7 v^{2}+b v^{2}+(u+v)^{2}\{(7+4 A) u+14 v\} k^{2}\right\} \lambda^{2}\right]
\end{gathered}
$$

\subsubsection{Nature of cosmological parameters}

In this RSII model, the deceleration parameter $q$ can be obtained as

$$
q^{(R S I I)}=-1-\frac{3}{2} \frac{\left\{\frac{\rho}{2 \lambda}\left(w_{m c g}^{(R S I I)} \frac{\rho_{m c g}}{\rho}-2\right)-\left(1+w_{m c g}^{(R S I I)} \frac{\rho_{m c g}}{\rho}\right)\right\}}{\left(1+\frac{\rho}{2 \lambda}\right)}
$$

which can be written in terms of dimensionless density parameter $\Omega_{m c g}=\frac{\rho_{m c g}}{\rho}$ as in the following

$$
q^{(R S I I)}=-1+\frac{3}{2} \frac{\left\{\left(\frac{-\rho}{2 \lambda}+1\right) w_{m c g}^{(R S I I)} \Omega_{m c g}+\left(1+\frac{\rho}{\lambda}\right)\right\}}{\left(1+\frac{\rho}{2 \lambda}\right)}
$$

Now since $\Omega_{m c g}=\frac{\rho_{m c g}}{\rho}=\frac{u}{u+v}$ and assuming $\frac{\rho}{\lambda}=\epsilon_{(R S I I)}$ we get,

$$
q^{(R S I I)}=-1+\frac{3}{2} \frac{\left\{\left(1-\frac{\epsilon_{(R S I I)}}{2}\right) w_{m c g}^{(R S I I)} \frac{u}{u+v}+\left(1+\epsilon_{(R S I I)}\right)\right\}}{\left(1+\frac{\epsilon_{(R S I I)}}{2}\right)}
$$

Considering only the first stable critical point, such that $(u, v) \rightarrow\left(u_{1 c}, v_{1 c}\right)$, using (49) we get,

$$
q_{c}^{(R S I I)}=-1+\frac{3}{2} X_{(R S I I)}, \quad \text { where } \quad X_{(R S I I)}=\frac{\left\{\left(1-\frac{\epsilon_{(R S I I)}}{2}\right) w_{m c g}^{(R S I I)} \frac{u_{1 c}}{u_{1 c}+v_{1 c}}+\left(1+\epsilon_{(R S I I)}\right)\right\}}{\left(1+\frac{\epsilon_{(R S I I)}}{2}\right)}
$$

If $\epsilon_{(R S I I)}=-\frac{2\left[\left(1+w_{m c g}^{(R S I)}\right) u_{1 c}+v_{1 c}\right]}{\left(2-w_{m c g}^{(R S I)}\right) u_{1 c}+2 v_{1 c}}, \quad X_{(R S I I)}=0, \quad$ we have $q=-1$, which confirms the accelerated expansion of the universe. When $\epsilon_{(R S I I)}=-2$ we have $q=-\infty$. Therefore we have super accelerated expansion of the universe.

In this scenario, the Hubble parameter can be obtained as,

$$
H=\frac{2}{3 X_{(R S I I)} t}
$$

where the integration constant has been ignored. Integration of (51) yields

$$
a(t)=a_{0} t^{\frac{2}{3 X(R S I I)}}
$$




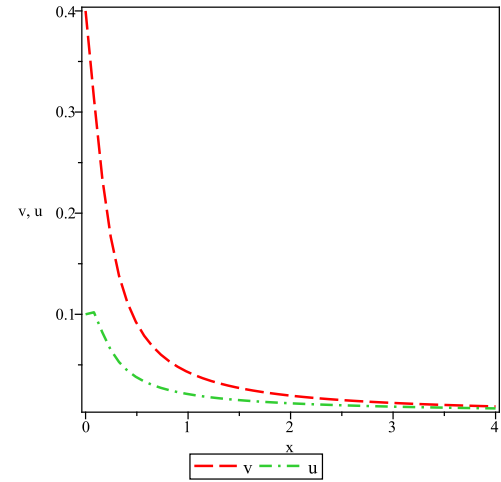

Fig. 8

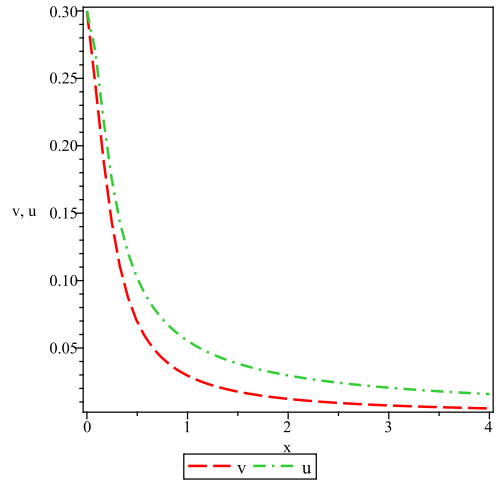

Fig. 9

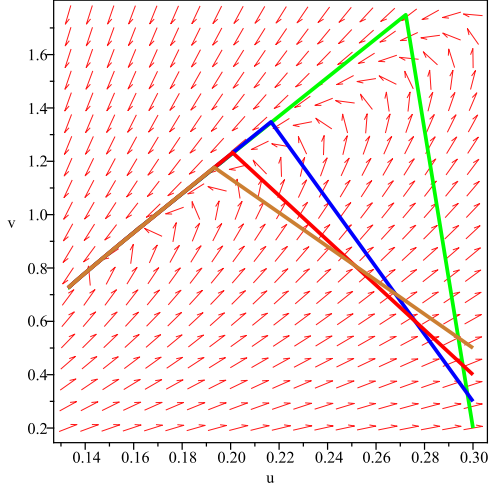

Fig. 10

Fig 8 : The dimensionless density parameters are plotted against e-folding time. The initial condition is $v(0)=0.4, u(0)=0.1$. Other parameters are fixed at $\alpha=1, b=0.5, A=0, B=1, \lambda=1$, and $\kappa=1$.

Fig 9 : The dimensionless density parameters are plotted against e-folding time. The initial condition is $v(0)=0.3, u(0)=0.3$. The other parameters are fixed at $\alpha=1, b=0.5, A=0, B=1, \lambda=1$ and $\kappa=1$.

Fig 10 : The phase diagram of the parameters depicting an attractor solution. The initial conditions chosen are $v(0)=0.2, u(0)=0.3$ (green); $v(0)=0.3, u(0)=0.3$ (blue); $v(0)=0.4, u(0)=0.3$ (red); $v(0)=0.5, u(0)=0.3$ (brown). Other parameters are fixed at $\alpha=1, b=0.01, A=0.3, B=1, \lambda=1$ and $\kappa=5$.

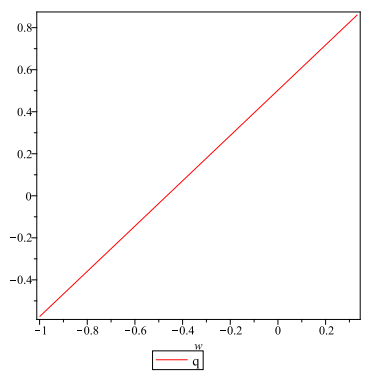

Fig. 11

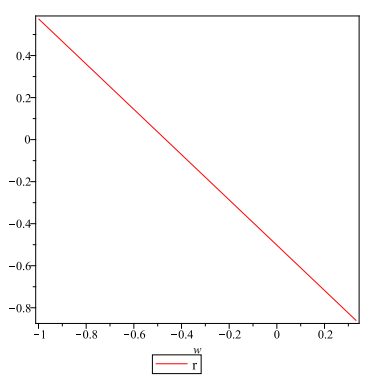

Fig. 12

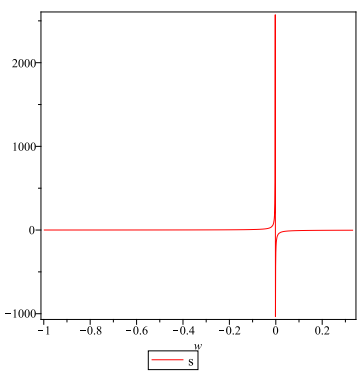

Fig. 13

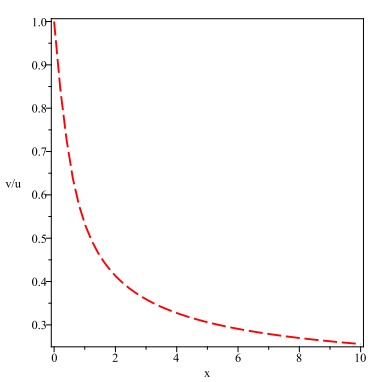

Fig. 14

Fig 11 : The deceleration parameter is plotted against the state parameter. $\epsilon=0.003$ is considered. Fig 12 : The statefinder parameter $r$ is plotted against the state parameter. $\epsilon=0.003$ is considered. Fig 13 : The statefinder parameter $s$ is plotted against the state parameter. $\epsilon=0.003$ is considered.

Fig 14 : The ratio of density parameters is shown against e-folding time. The initial conditions chosen are $v(0)=0.3, u(0)=0.3$. The other parameters are fixed at $\alpha=1, A=0, b=0.5, B=1, \lambda=1$ and $\kappa=1$. 
which gives the power law form of expansion of the universe. Like DGP brane here also in order to have an accelerated expansion of universe in RSII brane we must have $0<X_{(R S I I)}<\frac{2}{3}$. Using this range of $X_{(R S I I)}$ in the equation

$q_{c}^{R S I I}=-1+\frac{3}{2} X_{(R S I I)}$, we get the range of $q_{c}^{(R S I I)}$ as $-1<q_{c}^{(R S I I)}<0$. This is again consistent with an accelerated expansion of the universe as it was in DGP model. Like before here also we calculate the statefinder parameters $\{r, s\}$ in order to get relevant information of DE and DM in the context of background geometry only without depending on the theory of gravity. The expressions of the statefinder pair (eq. (26) ) in the RSII model can be obtained in the form

$$
r_{(R S I I)}=\left(1-\frac{3 X_{(R S I I)}}{2}\right)\left(1-3 X_{(R S I I)}\right) .
$$

and

$$
s_{(R S I I)}=X_{(R S I I)}
$$

\section{Graphical Representation of the Phase plane Analysis}

Phase diagrams are drawn to determine the type of critical point in DGP and RSII models. We discuss the results obtained in the two models separately.

\section{DGP BRANE MODEL}

The dimensionless density parameters $v$ and $u$ are drawn in figures 1 and 2. From the figures we see that $v$ decreases, and $u$ increases during evolution of the universe. This shows that the density of DM decreases while the density of DE increases as the universe evolves. So this result is consistent with the well known idea of an energy dominated universe. The phase space diagram (figure 3) shows the attractor solution. The eigen values are calculated at the critical point and found to be $(1.97625,-1.09495)$. Hence the critical point is a saddle point.

Figure 4 shows the variations of the deceleration parameter, $q$ against $w_{m c g}$. From figure 4 , it is evident that there is a gradual decrease in the deceleration parameter $q$, and finally in the late universe it attains negative values, which suggests that there should be an acceleration in the late universe. This result is in accordance with the various observational data of Ia supernovae and CMB data which suggests that the universe is undergoing an accelerated expansion of late. Figure 5 and 6 shows the variations of the statefinder parameters $r$ and $s$ against $w_{m c g}$. From these figures it can be seen that $r$ tends towards 1 and $s$ tends towards 0 . Therefore it is evident that these results tends towards the $\Lambda C D M$ model. Figure 7 shows the variation of the ratio of the density parameter $v$ and $u$ with time. From the figure it is evident that the ratio of the above parameters decreases with time. So it can be concluded that there is a relative decrease in matter density with respect to the energy density. This is again consistent with the notion of an energy dominated universe.

\section{RSII BRANE MODEL}

The dimensionless density parameters $v$ and $u$ in case of RSII brane model are drawn in figures 8 and 9 . From the figures it is evident that at early stages of evolution of universe density of DM decreases steeply relative to the density of DE such that finally an energy dominated scenario is witnessed in the late universe. The phase space diagram (figure 10) shows the attractor solution for the model. The eigen values are calculated at all the three critical points. At the first critical point the eigen values are found to be $(402.877,204.835)$. Hence the first critical point is an unstable node. At the second critical point the eigen values are found to be $(1214.75,565.097)$. Hence the second critical point is also an unstable node. At the third critical point the eigen values calculated are $(-25.3905+2257.49 i,-25.3905-2257.49 i)$. Hence the third critical point is a stable focus.

Figure 11 shows the behaviour of deceleration parameter $q$ as it is plotted against the state parameter $w_{m c g}$. The figure shows that there is a gradual decrease in the deceleration parameter with time. Finally in the late universe the deceleration parameter $q$ becomes negative, which confirms the fact that the universe is undergoing an accelerated expansion. In Figures 12 and 13, the statefinder parameters $r$ and $s$ are plotted against $w_{m c g}$. Finally in Figure 14 the ratio of the density parameters $v$ and $u$ is plotted against time. From the figure it is found that just like DGP brane here also there is a relative decrease in matter density with respect to the energy density. This is consistent with the notion of an energy dominated universe. Henceforth we find a solution for the cosmic coincidence problem. 


\section{$5 \quad$ Study of Future Singularities}

It is a well known fact that any energy dominated model of the universe is destined to result in a future singularity. The study of dynamics of an accelerating universe in the presence of DE and DM is in fact incomplete without studying these singularities, which are the ultimate fate of the universe. MCG, being a very generalized form of DE can step into the phantom era with the $\operatorname{EoS}(w<-1)$ for definite values of the constants $A$ and $B$. It is known that the universe dominated by phantom energy ends with a future singularity known as Big Rip (Caldwell, R. R., 2003), due to the violation of dominant energy condition (DEC). But this is not the only type of singularity possible. Nojiri et al (2005) studied the various types of singularities that can result from an phantom energy dominated universe. These possible singularities are characterized by the growth of energy and curvature at the time of occurrence of the singularity. It is found that near the singularity quantum effects becomes very dominant which may alleviate or even prevent these singularities. So it is extremely necessary to study these singularities and classify them accordingly so that we can search for methods to eliminate them. The appearance of all four types of future singularities in coupled fluid dark energy, $F(R)$ theory, modified Gauss-Bonnet gravity and modified $F(R)$ Horava-Lifshitz gravity was demonstrated in (Nojiri, S. and Odintsov, S. D., 2011). The universal procedure for resolving such singularities that may lead to bad phenomenological consequences was proposed. Nojiri, S. and Odintsov, S. D., 2011 classified and studied four types of such singularities in detail. We analyze two possible future singularities in case of MCG in brane world scenarios.

Type $I$ ("Big Rip") : For $t \rightarrow t_{s}, a \rightarrow \infty, \rho \rightarrow \infty$ and $|p| \rightarrow \infty$

Type II ("Sudden") : For $t \rightarrow t_{s}, a \rightarrow a_{s}, \rho \rightarrow \rho_{s}$ and $|p| \rightarrow \infty$

Here we have considered MCG as the DE in DGP and RS2 brane models. 1) Phantom era is realized for MCG when $\rho<\left[\frac{B}{A+1}\right]^{\frac{1}{\alpha+1}}$. From the MCG equation of state i.e $p=A \rho-\frac{B}{\rho^{\alpha}}$ we see that when $\rho \rightarrow \infty,|p| \rightarrow \infty$ when $a \rightarrow \infty$ and $t \rightarrow t_{s}$. Therefore it is seen that Type I singularity is quite obvious for MCG. This is the well known Big Rip singularity.

2) Suppose if $\rho \rightarrow \rho_{s}$ and $\rho_{s} \sim 0$, then $|p| \rightarrow-\infty$ for $t \rightarrow t_{s}$ and $a \rightarrow a_{s}$ Hence we get the type II singularity. This is called a sudden singularity.

The stability of the critical points analyzed in the last section will be occurred much before than the singularities take place, so any direct impact of the singularities of the dynamical system will not be observed. The big rip and sudden singularity may be formed before the critical points to be formed.

\section{Concluding Remarks}

In this work, we have considered a combination of Modified Chaplygin gas in DGP and RSII brane gravity models. Our basic idea was to study the background dynamics of MCG in detail when it is incorporated in brane gravity. Dynamical system analysis had been carried out, critical points were found and the stability of the system around those critical points was tested for both DGP and RSII brane models. Graphical analysis was done to get an explicit picture of the outcome of the work. In order to find a solution for the cosmic coincidence problem, a suitable interaction between DE and DM was considered. The dynamical system of equations characterizing the system was formed and a stable scaling solution was obtained. The numerical values of the density parameters for DE and DM almost coincided with each other (as seen in graphs 8 and 9). Hence this work can be considered to be a significant one as far as solution of cosmic coincidence problem is concerned. From the above analysis we conclude that the combination of MCG in brane gravity makes a perfect model for the expanding universe undergoing a late acceleration.

\section{Achknowledgement:}

RB thanks to State Govt. of West Bengal, India for awarding SRF.

\section{References}

Alam, U., Sahni, V., Saini, T. D., Starobinsky, A.A. :- MNRAS 344 1057(2003).

Alcaniz, J.S., Lima, J.A.S.:- Phys. Rev. D 72, 063516(2005).

Bamba, K., Matsumoto, J., Nojiri, S. :- arXiv : 1109.1308[hep-th].

Barreiro, T., Sen, A.A. :- Phys. Rev. D 70 124013(2004).

Barreiro, T.et. al.:- Phys. Rev. D $\mathbf{7 8} 043530(2008)$.

Benaoum, H. B.:- [arXiv:0205140(hep-th)]. 
Bento, M. C., Bertolami, O., Sen, A. A. :- Phys. Rev. D 66, 043507(2002).

Berger, M.S., Shojae, H.:- Phys.Rev.D 73,083528(2006).[ arXiv:0601086(gr-qc)].

Bouhmadi-Lopez, Lazkoz, R.:- Phys. Lett. B 654, 51(2007) [arXiv:0706.3896 (astro-ph)].

Brax, P. et. al :- Rep. Prog.Phys. 67, 2183(2004)[arXiv:0404011(hep-th)].

Caldwell, R.R., Kamiokowsky,M., Weinberg,N.N. :- Phys. Rev. Lett. 91071301 (2003).

Carturan, D., Finelli, F. :-Phys. Rev. D 68 103501(2003).

Chimento,L. P.,Lazkoz, R.,Maartens, R., Quiros, I. :- JCAP 0609, 004(2006)[arXiv:0605450(astro-ph)].

Csa ki, C.:- [arXiv:0404096(hep-th)].

Dalal, N., Abazajian, K., Jenkins, E., Manohar, A.V., :- Phys. Rev. Lett 141302(2001) arXiv:astro-ph/0105317v1].

Dao-Jun, L., Xin-Zhou, L. :- Chin. Phys. Lett. 22 1600(2005).

Debnath, U., Banerjee, A., Chakraborty, S. :- Class. Quantum. Grav. 21, 5609(2004).

Debnath, U. :- Astrophys. Space Sci. 312, 295 (2007).

Deffayet, D.:- Phys.Lett. B 502 199(2001).

Deffayet, D., Dvali, G.R., Gabadadze, G.:-Phys.Rev.D 65044023 (2002)[arXiv:0105068(astro-ph)].

del Campo, S., Herrera, R., Pavon, D. :- JCAP 0901 020(2009).[arXiv:0812.2210 (gr-qc)].

Dev, A.,Alcaniz, J. S., Jain, D. :- Phys. Rev. D. 67 , 023515(2003).

Dvali, G. R. , Gabadadze, G., Porrati, M.:- Phys.Lett. B 485 208(2000)[arXiv:000506(hep-th)].

Feng, C. et. al.:- Phys. Lett. B 665, 111(2008).

Gorini, V., Kamenshchik, A. and Moschella, U. Pasquier, V. :- [arXiv:0403062(gr-qc)].

Gorini, V., Kamenshchik, A. and Moschella, U. :- Phys. Rev. D 67, 063509(2003).

Griest, K., Phys.Rev.D66:123501(2002).[arXiv:0202052].

Guo, Z.-K., Zhang, Y.-Z.:- Phys. Rev. D 71, 023501 (2005).

Hu, B., Ling, Y. :- Phys. Rev. D 73 123510(2006).

Jamil, M., Rahaman, F.:- Eur. Phys. J. C 64 97(2009).

Jamil, M., Saridakis, E.N., Setare, M.R. :- Phys. Rev. D 81 023007(2010a).

Jamil, M., Saridakis, E.N. :- JCAP 07 028(2010b).

Jamil, M., Farooq, M.U.:- JCAP 03 001(2010c).

Jamil, M., Sheykhi, A., Farooq, M.U.:-Int. J. Mod. Phys. D 19 1831(2010d).

Jamil, M. :- Int. J. Theor. Phys. 49 62(2010e).

Jamil, M., Momeni, D., Rashid, M.A., :- Eur. Phys. J.C 71 1711(2011) arXiv:1107.1558v1 [physics.gen-ph]].

Jimenez, J.B., Maroto, A.L.:- AIP Conf.Proc. 1122,107(2009).[arXiv:0812.1970 (astro-ph)].

Jing, H. et. al.:- Chin. Phys. Lett. 25 347(2008).

Kamenshchik, A., Moschella, U. and Pasquier, V. :- Phys. Lett. B 511, 265(2001).

Leon, G., Saridakis, E.N.:- Phys.Lett.B 693 1(2010).[ arXiv:0904.1577 (gr-qc)].

Lu, J. et. al., Phys. Lett. B 662 (2008) 87.

Lue,A., Starkman,G. D. :-Phys. Rev. D 70, 101501(2004) [arXiv:0408246(astro-ph)].

Maartens, R., Phys. Rev. D 62084023 (2000).

Maartens, R.:-Living Rev. Relativity 7 7(2004)[arXiv:0312059(gr-qc)].

Maeda, K. and Wands, D., Phys. Rev. D 62124009 (2000).

Makler, M. et. al.:- Phys. Lett. B 555 1(2003).

Nojiri, S., Oditsov, S.D. :- Phys. Lett. B 571 1(2003).

Nojiri, S., Oditsov, S.D. :- Phys. Rev. D 70 103522(2004).

Nojiri, S., Oditsov, S.D., Tsujikawa, S. :- Phys. Rev. D 71063004(2005).

Nojiri, S. and Odintsov, S. D.:- Phys. Rept. 50559 (2011). Panotopoulos, G. :- Phys. Rev. D $77107303(2008)$.

Perlmutter, S. et al. :- [Supernova Cosmology Project Collaboration], ApJ 517, 565(1999) [arXiv:astro-ph/9812133].

Randall, L., Sundrum, R.:-Phys. Rev. Lett. 83, 3770(1999a).

Randall, L., Sundrum, R.:-Phys. Rev. Lett. 83, 4690(1999b).

Riess, A. G. et al. :- [Supernova Search Team Collaboration], ApJ 607, 665(2004)[arXiv:0402512(astro$\mathrm{ph})$.

Rubakov, V. A.:-, Phys. Usp.44, 871(2001) [arXiv:0104152(hep-th)].

Sahni, V. et al.:- JETP 77, 201(2003).

Sasaki, M., Shiromizu, T. and Maeda, K., Phys. Rev. D 62024008 (2000.

Setare, M. R. :- Phys.Lett B 642 1(2006).

Setare, M. Bouhmadi-Lopez, M.R. :- Phys.Lett B 648, 329 (2007a). 
Setare, M. R. :- Eur. Phys. J. C 52 689(2007b).

Setare, M. R. :- Eur. Phys. J. C 50 991(2007c).

Setare, M. R. :- IJMPD 18 419(2009).

Shiromizu, T., Maeda, K. and Sasaki, M., Phys. Rev. D 62024012 (2000).

Spergel, D. N. et al. :- WMAP Collaboration,Astron. J. Suppl 148, 175(2003) [arXiv astro-ph/0302209].

Thakur, P. , Ghose, S. , Paul, B. C.:-MNRAS 397, 1935 (2009).

Wu, P., Yu, H. :- Class. Quant. Grav. 24 4661(2007).

Wu, X., Cai, R. G., Zhu, Z. H. :- Phys.Rev.D 77,043502(2008).

Zhang, H., Zhu, Z. H. :- Phys.Rev.D 75,023510(2007).

Zhang, X.:- Mod.Phys.Lett.A 20 2575(2005). 